\title{
Narrativas de la vulnerabilidad: periodistas juarenses frente a la cobertura informativa de la COVID-19
}

\section{Narratives of vulnerability: journalists from Juarez in the informative coverage of the COVID-19}

María Cristina Tamariz Estrada* Escuela de Periodismo Carlos Septién García Basilio Vadillo núm. 43, Col. Tabacalera, C.P. 06030, Ciudad de México, México

Editor: Rogelio del Prado Flores

Fecha de recepción: 6 de julio de 2020

Fecha de aceptación: 22 de julio de 2020 xtina.tamariz@gmail.com https://orcid.org/0000-0002-7014-1998

\section{RESUMEN}

https://doi.org/10.36105/stx.2020edespcovid-19.03

El artículo presenta los primeros hallazgos de un estudio exploratorio de corte cualitativo sobre las experiencias de los periodistas en la cobertura informativa por COVID-19 en Ciudad Juárez. A partir de entrevistas semiestructuradas se analiza de manera comparativa los procesos de planeación, el rol del periodismo en contextos de emergencia, así como los impactos (laborales y emocionales) de la cobertura en un periódico impreso, El Heraldo de Juárez y otro digital, Norte. Los resultados muestran que en contextos de emergencia: a) el uso de plataformas virtuales para trabajo a distancia es indispensable en la organización del trabajo editorial, b) las restricciones de distanciamiento afectan el trabajo periodístico y el intercambio directo con las autoridades, c) en el contexto de la crisis sanitaria, los periodistas se muestran preocupados ante la incertidumbre laboral, el riesgo a su salud y los impactos emocionales de la cobertura.

Palabras clave: periodismo, cobertura, CovID-19, Ciudad Juárez.

\footnotetext{
* Investigadora y docente en temas de comunicación y prácticas periodísticas. Especialista en diseño de investigación aplicado al periodismo, forma parte del cuerpo docente de la Maestría en Periodismo Político de la Escuela de Periodismo Carlos Septién García desde el 2016. Pertenece al Sistema Nacional de Investigadores (SNI). Es licenciada en Comunicación y Periodismo por la UNAM; maestra es Sociología Política por el Instituto Mora y doctora en Ciencias Sociales con mención en Sociología por el Colegio de México.
} 


\section{ABSTRACT}

The article presents the first findings of a qualitative exploratory study on the experiences of journalists in the news coverage by COVID-19 in Ciudad Juárez. From semi-structured interviews, the planning processes, the role of journalism in emergency contexts, as well as the impacts (labor and emotional) of coverage in a printed newspaper, El Heraldo de Juárez and another digital, Norte, are analyzed in a comparative way. The results show that in emergency contexts: a) the use of virtual platforms for remote work is essential in the organization of editorial work, b) distance restrictions affect journalistic work and direct exchange with the authorities, c) In the context of the health crisis, journalists are concerned about job uncertainty, the risk to their health and the emotional impacts of coverage.

Keywords: journalism, coverage, CoviD-19, Ciudad Juárez.

\section{INTRODUCCIÓN}

a cobertura periodística de emergencias y desastres incluye fenómenos de Utipo geofísico, meteorológico y biológico que afectan a una población vulnerable. A principios del siglo xx se incluían como parte de estos fenómenos epidemias, inundaciones, sequías y hambrunas. En la actualidad, desde la perspectiva de la protección civil los fenómenos perturbadores se clasifican en geológicos, hidrometereológicos, químico-tecnológicos, socio-organizativos y sanitario-ecológicos (Segob \& Cenapred, 2006). En esta última categoría están las plagas, la contaminación del aire, agua y suelo y lo que nos ocupa, las epidemias.

En su conjunto, todos los fenómenos señalados son materia de lo que se conoce como periodismo de desastres, no obstante, cada uno delimita un conjunto de posibilidades y restricciones en su cobertura informativa. Por definición, los desastres enfrentan a las poblaciones a peligros y cambios en sus vidas y actividades, implican reacciones ante la crisis y suponen ajustes con los cuales las personas resuelven problemas cotidianos de riesgo y vulnerabilidad. En estos puntos se centra también la atención periodística cuando una epidemia a gran escala tiene lugar (Reyna, 1998).

Las coordenadas del inicio de la epidemia por coronavirus señalan una fecha: diciembre de 2019 y un lugar, Wuhan, China. A partir de ese momento una suce- 
sión de contagios importados configuró las condiciones para que el brote epidémico tuviera un impacto mundial y fuera declarado pandemia por la Organización Mundial de Salud (oms). El seguimiento informativo centró su atención inicialmente en China, después en Italia y España, respectivamente. El nuevo virus llegó a Estados Unidos el 21 de enero de 2020 y a México el 27 de febrero. Para el 11 de marzo, la oms declaró pandemia al brote de nuevo coronavirus.

En México, en la ciudad fronteriza de Juárez, la cronología de la epidemia se activó con casi un mes de diferencia. Ante la llegada inminente del virus, las autoridades del estado de Chihuahua encabezadas por el gobernador Javier Corral y sus funcionarios de salud, iniciaron una campaña de información preventiva desde el 22 de enero, un día después de que Estados Unidos anunciará su primer caso. En Juárez el primer caso se detectó el 17 de marzo, un individuo de 29 años que reportó viaje a Italia. En esa fecha, las autoridades federales de salud declaraban la entrada en la fase 2 con un total de 100 casos confirmados en el país.

En Ciudad Juárez, los medios locales preparaban algunas medidas para concentrar la cobertura informativa en la CoviD-19, entre las que destaca el trabajo a distancia. Sin embargo, para quienes están en campo, reporteros y fotógrafos, el desafío sería mayúsculo porque los enfrentaba a una situación de riesgo inédita que, en comparación con las crisis anteriores, por violencia y temas migratorios, comprometía su salud directamente.

Para entender la dinámica de la cobertura informativa de las epidemias, recurrimos al concepto de los ciclos mediático-epidémicos, retomado por Waisbord de Shih, Wijaya y Brossard (2008), el cual explica en tres momentos la dinámica del tema, en este caso covid-19, en la agenda periodística (ver Esquema 1). Se reconoce que el comportamiento y avance de la epidemia, con estadísticas nuevas a diario alimenta el seguimiento noticioso. Sumado a lo anterior, el riesgo generalizado por la proximidad geográfica y social acentúan el interés periodístico.

En términos del ejercicio reporteril, Waisbord apunta que la cobertura de las epidemias se puede mantener con recursos mínimos aún en las condiciones de precarización laboral que distinguen al periodismo en América Latina. Es suficiente con el seguimiento de las estadísticas, entrevistas a personal médico, crónicas de poblaciones vecinas y declaraciones de altos funcionarios, cuando el tema político domina el ciclo noticioso. Es probable que, gracias a esa relativa facilidad para cubrir brotes epidémicos, las condiciones de riesgo y vulnerabilidad que asumen los reporteros queden invisibilizadas. Al respecto, consideramos que es necesario problematizar el argumento y explorar a partir de las prácticas 
y experiencias de los reporteros, los desafíos que enfrentan en lo profesional y en lo personal con la cobertura informativa de una epidemia.

\section{ESQUema 1. CiClos mediátICO-EPIdÉMICOS}

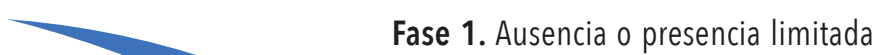
de información sobre la epidemia en secciones especiales.

Fase 2. Duración prolongada y priorización en el ciclo noticioso. Momento cumbre, los problemas de salud pasan a conflictos políticos y decisiones ciudadanas. Domina la narrativa de riesgo.

Fase 3. Regreso a la cobertura mínima. Por respuesta eficaz del sistema de salud o por una decisión oficial de no dar más información sobre el tema.

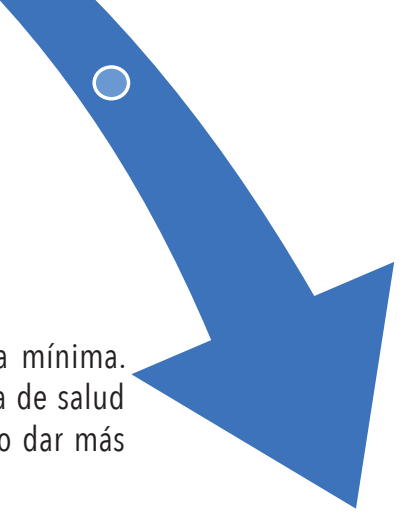

FUENTE: ELABORACIÓN PROPIA CON BASE EN WAISBORD, 2010.

En ese contexto, las preguntas de investigación que orientan este estudio exploratorio se concentran en exponer las experiencias de los periodistas juarenses en el contexto de la emergencia sanitaria por Covid-19. Las preguntas orientadoras son: ¿cómo se planeó la reorganización del trabajo editorial y reporteril ante la pandemia?, para los reporteros, ¿cuál es el papel del periodismo local en un contexto de emergencia?, ¿qué desafíos enfrentan en la cobertura informativa por CoviD-19? y ¿cuál es la percepción de vulnerabilidad de los periodistas ante la epidemia? ${ }^{1}$

1 Márquez y Hughes (2016) señalan la escasez de estudios que profundicen en cómo los periodistas asumen y perciben su realidad laboral, profesional y política. Entre sus conclusiones sobre el perfil demográfico y profesional de los periodistas en México, señalan que en su mayoría cuentan con estudios universitarios, tienen una edad promedio de 38 años, lo cual habla del conocimiento y experiencia necesarios para el ejercicio de la profesión. Sin embargo, detectan condiciones de 
Cada una de las preguntas se plantea como un primer acercamiento por medio de entrevistas a periodistas de El Heraldo de Juárez y Norte Digital. El primero, un medio impreso de corte tradicional, el segundo transitó a formato digital en abril de 2017, después del asesinato de la periodista Miroslava Breach. Los ejes que articulan este primer acercamiento son: los procesos de planeación, el rol del periodismo en contextos de emergencia, así como los impactos (laborales y emocionales) de la cobertura covid-19 en Ciudad Juárez, en el periodo del 28 de abril al 20 de mayo.

\section{DESARROLLO}

\section{Un acercamiento al periodismo de desastres}

El tema de los desastres y emergencias en medios puede dividirse entre los estudios orientados al análisis de los contenidos informativos y en otra vertiente, lo que se conoce como periodismo de desastres, es decir, a las prácticas que los periodistas desarrollan ante la cobertura de fenómenos que ponen en riesgo a la población. En este rubro encontramos una diversidad de situaciones, desde atentados, inundaciones, guerras, sismos, por lo que sería difícil llegar a una conclusión definitiva al respecto, en tanto cada situación exige un abordaje particular.

Desde esta perspectiva, sobresalen las investigaciones que desde las no-rutinas describen los procesos de adaptación y las estrategias que los periodistas implementan en la cobertura de los desastres, al dimensionar los alcances de la situación a cubrir y considerar los antecedentes para solucionar en lo inmediato las posibles complicaciones en materia de recopilación de información (Berkowitz, 1992; Olsson, 2010; Domíngez-Panama, 2017).

Una de las principales críticas al ejercicio periodístico en la cobertura de desastres está en el énfasis en la espectacularización de la tragedia, el consumo de imágenes de alto impacto sin un abordaje a profundidad sobre las causas del fenómeno. La atención en lo inmediato, sin documentar antecedentes ni dar seguimiento, priva al público de conocer el desenlace. Ante la "fatiga de la compa-

precarización laboral por salarios bajos en relación con la carga de trabajo. El punto que rescatamos a propósito de sus condiciones laborales es la dificultad que tienen para especializarse en algún rubro, si pensamos en el periodismo de desastres, es importante considerar este tipo de perfiles por el grado de exigencia y formación que exige la cobertura de emergencias y desastres. 
sión”, el público pierde interés en información repetitiva de la cual desconoce sus alcances (Toledano \& Ardèvol-Abreu, 2013).

Como reconocen Rodríguez y Odriozola (2012), referirse al periodismo de catástrofes involucra una especialidad compleja que exige abordar el hecho noticioso, las afectaciones del fenómeno, los impactos materiales y emocionales. Pero involucra a su vez otros ámbitos como parte de los efectos como el político, económico y, en el caso de las epidemias, sanitario. Lo anterior no significa un desacuerdo con los lineamientos éticos a seguir en estos casos, la dinámica de cada emergencia es particular desde la experiencia de los periodistas en su cobertura.

Una alternativa para trascender la inmediatez que caracteriza las coberturas de desastres parte de la misma lógica de la gestión del riesgo, es decir, de la prevención. Anticiparse al desastre, plantear distintos escenarios ante la emergencia de posibles fenómenos disruptivos. Documentar los antecedentes de los puntos clave en la cobertura de las emergencias, proporcionan a los periodistas perspectiva para elaborar contenidos en donde se discutan las causas y efectos posibles de los desastres (Bernabé, 2007).

Otro punto sensible en la cobertura de desastres tiene que ver con los impactos emocionales a los que se exponen los periodistas. Por lo general, para los responsables de los medios es un aspecto menor, aunque las investigaciones documenten lo contrario. El manejo de las emociones por parte de los periodistas que recopilan los testimonios de las víctimas y describen los escenarios de la tragedia implica un punto de equilibrio para la autoprotección física y emocional sin perder la sensibilidad y empatía que exige el trato con los afectados (Camps, 1999).

Es común, según Hight y Smyth (2003), que los periodistas presenten los mismos síntomas de estrés traumático que otros profesionales, como son policías, bomberos, médicos, que están en la primera línea de atención en situaciones de desastre o emergencia. La diferencia es que estos impactos son invisibilizados, no hay reconocimiento ni la atención adecuada para su contención, "mientras a los trabajadores de seguridad pública se les ofrece sesiones informativas y orientación después de la tragedia, a los periodistas se les asigna la cobertura de otra historia" (2003, p. 3).

Documentar la experiencia de los periodistas en coberturas de desastre nos permitirá problematizar todos los elementos que afectan en el seguimiento informativo de estos temas. También aportará elementos para lograr que el papel social del periodismo en situaciones de emergencia cumpla su cometido como 
servicio público de primer orden optimizando las condiciones de trabajo de los profesionales de la información.

En el siguiente apartado hacemos un breve repaso de los periódicos seleccionados para este estudio.

\section{BREVE RECUENTO DE DOS MEDIOS PERIODÍSTICOS EN CIUDAD JUÁREZ}

En su caracterización del panorama mediático de Ciudad Juárez, Checa Godoy (2008) distingue a la prensa juarense por el número reducido de periódicos en comparación con otras ciudades con características similares, así como en su influencia por el número de lectores; sus índices de lectura son superiores al de los estados, incluyendo a la Ciudad de México. En 1976 surge uno de los periódicos influyentes en la actualidad, El Diario de Juárez, reconocido también como uno de los más leídos en el país e identificado con el Partido Revolucionario Institucional (PRI). En 1990, el corporativo periodístico Norte llegaría a competir directamente con El Diario de Juárez, ofreciendo a sus lectores investigaciones sobre corrupción y reportajes con un mayor grado de elaboración. Además, influye en el bipartidismo político presente en Chihuahua, al dar voz a la oposición emergente, principalmente del Partido Acción Nacional (PAN). A continuación, se presenta una breve descripción y cronología de los medios que forman parte del presente estudio.

\section{El Heraldo de Juárez}

Los antecedentes de El Heraldo de Juárez se remontan al año de 1947, con el surgimiento del periódico El Mexicano, que en un principio pertenecía a la cadena García Valseca y después formó parte de la Organización Editorial Mexicana (OEM). El Mexicano era identificado como el tercer periódico en importancia en Ciudad Juárez, asociado a los viejos esquemas del periodismo sensacionalista, según explica Checa Godoy, "enmascara todo el drama de los feminicidios como la crónica de sucesos cotidiana" (2008, p. 203).

En 2019 tiene lugar una reorientación del periódico, a decir del subdirector editorial, Francisco Cabrera. La estrategia para el relanzamiento fue cambiar el nombre a El Heraldo de Juárez y romper con el estigma del corte policíaco en sus contenidos: "Yo entré hace dos años a la empresa y se decidió que el periódico era 
matutino y por cuestión de negocios y todo eso, le cambiamos el nombre, porque la marca El Mexicano nos cerraba puertas, y una vez que lo cambiamos fue mejorando" (Entrevista Cabrera, 2020).

En los últimos años, la oEM entró en un proceso de reestructuración y control de sus editoras. Actualmente tiene 46 periódicos en todo el país. Hace un par de años modificó el sistema de producción de los periódicos. Según explica Cabrera, el uso de un software llamado internamente CCI optimiza los tiempos al permitir trabajar en nube. El trabajo editorial se organiza a través de mesas de redacción regionales. Los editores tienen acceso a los contenidos en tiempo real de los periódicos que pertenecen a su región. En el contexto de la emergencia sanitaria, las actividades de reporteo son las que sufrieron cambios sustantivos como se verá más adelante.

\section{Norte Digital}

El periódico Norte publica su primera edición impresa el 6 de mayo de 1990, bajo la dirección de Óscar Cantú Murguía, empresario juarense que, en octubre de 1981, en sociedad con Juan Francisco Ealy Ortiz, Jesús Macías Delgado, Rómulo Escobar y Arturo Quiroz, publicaron El Universal de Ciudad Juárez. Desde sus inicios en Norte, las amenazas e intimidación de sus periodistas ha sido una constante frente a las investigaciones que en su momento denunciaban los vínculos de autoridades locales con el crimen organizado.

Las disputas electorales también fueron el escenario propicio para condicionar la compra de publicidad y afectar así la viabilidad del proyecto en represalia por abrir el espacio periodístico a los candidatos panistas. Entre reportajes de denuncia a las autoridades municipales y del estado se sucedieron episodios de intimidación y amenazas por concepto de incumplimiento de publicidad oficial contratada. En 2014, tras la publicación de un reportaje que involucraba al gobernador y su relación con una institución bancaria, el gobierno del estado canceló los contratos de publicidad. La llegada del panista Javier Corral en octubre de 2016 no mejoró los pronósticos de viabilidad económica del periódico, en tanto se negó a saldar los servicios contratados por su antecesor, así como los compromisos adquiridos en ese rubro por su administración los primeros meses de gobierno.

Finalmente, el 2 de abril de 2017, el dueño de Norte firmaba una carta en la que anunciaba el cierre de su edición impresa, a unos días del asesinato de Miroslava Breach, ocurrido el 23 de marzo. En la carta de despedida, Cantú se refería a dos 
factores que lo llevaron a tomar la decisión del cierre: el alto riesgo que enfrentan los periodistas que costó la vida de una de sus colaboradoras y "el irresponsable incumplimiento de las administraciones públicas en los tres niveles de Gobierno también nos orilla a tomar esta decisión, ante la soberbia negativa de pagar adeudos contraídos por la prestación de servicios". Pasaría poco más de un año para que el 1 de abril de 2018, Cantú anunciara el regreso de Norte en una edición quincenal libre de publicidad oficial. En febrero de 2019 la periodicidad cambió a semanal, el 30 de marzo se publicó la última versión impresa de Norte, por lo cual a partir de ese momento el medio se concentró en el portal, Norte Digital.

\section{Abordaje metodológico}

La propuesta metodológica del presente estudio exploratorio parte de un acercamiento cualitativo a las experiencias de los periodistas de dos medios en Ciudad Juárez, El Heraldo de Ciudad Juárez y Norte Digital durante la cobertura sobre Covid-19. El criterio de selección de los medios elegidos, uno impreso y otro digital, fue el de tener una perspectiva comparada. Para contactar desde la Ciudad de México a los posibles entrevistados aproveché mi red de contactos con exalumnos de posgrado de la Universidad Autónoma de Ciudad Juárez, en su totalidad periodistas en activo en diversos medios de la ciudad. La estrategia para la realización de las entrevistas incluía además de reporteros, un acercamiento con responsables del trabajo editorial para conocer cómo se planeó la cobertura y cómo impactaron estas decisiones en la labor de reporteo. A continuación, se presenta el perfil de los periodistas entrevistados. Las entrevistas se realizaron por vía Skype entre el 28 de abril y el 20 de mayo de 2020.

PERFIL ENTREVISTADOS COBERTURA COVID-19

EN JUÁREZ (ABRIL-MAYO 2020)

\section{El Heraldo de Juárez}

- Francisco Cabrera (fecha de entrevista: 28/04/2020). Subdirector de El Heraldo de Juárez. Presidió en 2015 la mesa directiva de la sociedad de Periodistas y Comunicadores de ciudad Juárez. Ingresó a El Heraldo de Juárez en 2018. 
- Paola Gamboa (fecha de entrevista: 09/05/2020). Reportera con ocho años de experiencia. Actualmente trabaja para el periódico El Heraldo de Juárez. Desde los últimos cinco años dedicada a cubrir salud, tema migrante. Actualmente en El Heraldo de Juárez cubre al cien por ciento COVID-19.

\section{Norte Digital}

- Guadalupe Salcido (fecha de entrevista: 26/04/2020). Responsable editorial. 23 años de trayectoria en el periódico El Norte, hasta el cierre de la edición impresa en 2017. Regresa como colaboradora de Norte Digital, contratada para trabajar el tema de la pandemia de la Covid-19, 2020.

- Carlos Barranco (fecha de entrevista: 09/05/2020). En 1993 egresó de la Universidad Veracruzana. Ganador de la Columna de plata y mención honorífica en el premio Breach Valdez de 2019. Trabaja en Norte desde 2015.

- Luis Villagrana (fecha de entrevista: 20/05/2020). Estudió Trabajo Social y Sociología en la Universidad Autónoma de Ciudad Juárez. Entró al Diario de Juárez en 1987. Ha trabajado en varias etapas en Norte, en total ocho años. Reingreso a Norte Digital en octubre de 2019.

\section{CONTEXTO Y EVOLUCIÓN DE LA PANDEMIA \\ EN EL ESTADO DE CHIHUAHUA}

Desde el inicio de la epidemia en Chihuahua, Ciudad Juárez concentró la mayoría de casos positivos y defunciones por Covid-19. El contexto sanitario en el que se realizaron las entrevistas atiende a la segunda etapa del 'ciclo mediático-epidémico', caracterizada por mover el foco de atención del tema de salud a los conflictos políticos derivados de las decisiones de autoridades con respecto a la epidemia. Además, está presente una narrativa de riego que expone "la idea de un mundo fuera de control, presenta una situación donde la normalidad de la presunta estabilidad y orden es quebrada por enfermedades que vulneran los sistemas de vigilancia y control epidémico" (Waisbord, 2010, p. 100). Esta narrativa quedará expuesta a detalle en el análisis de las entrevistas.

En la siguiente tabla se puede observar el comportamiento de la epidemia desde la detección del primer caso en la entidad, el 17 de marzo, hasta el periodo 
que comprende la realización de las entrevistas, 28 de abril al 20 de mayo. El número de casos positivos pasó de 289 el 30 de abril (dos días después de la primer entrevista), a 520 a finales de mayo, cuando tuvo lugar la última entrevista del estudio. Con las defunciones sucedió lo mismo, el 30 de abril se registraban 73 y el 30 de mayo, 260. En ese contexto de aumento exponencial de casos y defunciones se realizaron las entrevistas, en la etapa 2 del ciclo mediático-epidémico definido por Waisbord. El compartamiento de la epidemia estaba lejos de estabilizarse ya que al concluir el estudio, a finales de junio el número de defunciones, 502 al día 26 , casi se habían duplicado con respecto al mes de mayo, mientras que la cifra de casos positvos era de 2595 .

\begin{tabular}{|l|c|c|c|c|}
\hline FECHA & $\begin{array}{c}\text { CASOS POSITIVOS } \\
\text { EN MUNICIPIOS DE } \\
\text { CHIHUAHUA }\end{array}$ & $\begin{array}{c}\text { CASOS POSITIVOS } \\
\text { EN CIUDAD JUÁREZ }\end{array}$ & $\begin{array}{c}\text { DEFUNCIONES EN } \\
\text { MUNICIPIOS DE } \\
\text { CHIHUAHUA }\end{array}$ & $\begin{array}{c}\text { DEFUNCIONES EN } \\
\text { CIUDAD JUÁREZ }\end{array}$ \\
\hline $\mathbf{1 7}$ de marzo & - & 1 & - & - \\
\hline $\mathbf{3 0}$ de marzo & 6 & 4 & 22 & 19 \\
\hline $\mathbf{1 6}$ de abril & 117 & 82 & 86 & 73 \\
\hline $\mathbf{3 0}$ de abril & 451 & 400 & 98 & 80 \\
\hline $\mathbf{1 0}$ de mayo & 600 & 520 & 322 & 260 \\
\hline $\mathbf{3 0}$ de mayo & 1187 & 2595 & 634 & 502 \\
\hline $\mathbf{2 6}$ de junio & 4355 & 289 & - & 73 \\
\hline
\end{tabular}

FUENTE: Evolución de casos positivos y defunciones en Ciudad Juárez. Elaboración propia con cifras de la Secretaría de Salud del Estado de Chihuahua.

En ese contexto de inicio de la epidemia, los entrevistados dieron cuenta de las primeras impresiones a propósito de la cobertura. A continuación, se exponen los hallazgos principales del estudio. 


\section{ANÁLISIS DE LAS ENTREVISTAS}

Los primeros hallazgos del presente estudio de tipo exploratorio con orientación metodológica cualitativa se organizan a partir de los siguientes ejes temáticos:

- Preparación de la cobertura. Incluye la reorganización del trabajo editorial y la producción de contenidos en el contexto de la emergencia.

- Los desafíos de la cobertura con énfasis en los cambios en las rutinas de trabajo de los reporteros, el acceso y restricciones a las fuentes de información y el rol del periodismo ante la emergencia sanitaria.

- Los impactos de la cobertura. Percepción de vulnerabilidad y riesgo y estrategias para hacer frente a los impactos del trabajo periodístico en el contexto de la pandemia por covid-19.

\section{Preparación de la cobertura}

La preparación de la cobertura informativa por CoviD-19 en Ciudad Juárez tuvo como antecedente las conferencias de prensa que el gobierno del estado inició a mediados del mes de enero. De carácter preventivo, estos espacios alertaron a medios y sociedad sobre las medidas de protección a seguir una vez que el primer caso fuera detectado. Ese momento llegó el 17 marzo, es decir con tres semanas de diferencia con respecto al primer caso en el país (27 de febrero). En los medios, las decisiones para resolver la cobertura de la epidemia coincidieron en aspectos como el trabajo a distancia, en sincronía con las medidas de protección promovidas por las autoridades locales y federales.

En El Heraldo de Juárez, las primeras pruebas para el trabajo a distancia iniciaron el 27 de marzo, es decir, dos fines de semana antes del inicio de la fase 2. Para el trabajo diario entre reporteros y dirección editorial, se decidió utilizar herramientas de Google para el trabajo editorial que permitían tener reuniones virtuales, compartir documentos y realizar videollamadas. Por parte de la empresa, la orden fue mandar al 80\% de la plantilla a laborar desde casa. Quedaron solo los distribuidores responsables de llevar el periódico a los puntos de venta. En la oficina se mantuvieron solo guardias de dos personas del equipo administrativo. Las nuevas rutinas del trabajo a distancia arrojaron resultados positivos con respecto a los tiempos de entrega para el cierre de la edición, como lo explica Francisco Cabrera: 
En estos momentos ninguno de nuestros reporteros va a las oficinas. Nos reunimos a las 8 de la mañana a través de Google, Hangouts. Con esta nosotros hacemos las videollamadas y ahí damos las órdenes como si estuvieras en la oficina. Ahí cada uno de los colaboradores aporta, hace sus comentarios, revisamos la edición, etc. Luego, a la 1 de la tarde tenemos otra reunión que es una mesa regional con los directivos, editores de Chihuahua, de Parral, para ver los temas nacionales y ahí pedimos información. Después, a las 2 ya tenemos otra reunión para planear nuestra edición de El Heraldo y ya de ahí nada más estamos checando la plataforma que te comentaba (Entrevista Cabrera).

El trabajo a distancia entra en conflicto con el ejercicio de reporteo, como reconoce Cabrera, "finalmente somos contadores de historias, es lo que hacemos y las historias están en la calle". En este medio, la cobertura se agregó un producto editorial adicional, el suplemento COVID, en formato tabloide.

Por otra parte, en Norte Digital también iniciaron de manera temprana con una sección especial llamada "Coronavirus", en principio alimentada con notas informativas sobre el tema en otros países y con las primeras conferencias de prensa del gobierno de Chihuahua. En este medio digital, el trabajo a distancia se consolidó cuando la empresa planteó la posibilidad de que tanto reporteros, editores, fotógrafos y todos lo que tienen participación en el diseño editorial y de contenidos se llevaran el equipo a sus domicilios y organizar el trabajo diario por medio de juntas virtuales. Carlos Barranco explica cómo se definió la cobertura COVID-19 con respecto a los formatos y al uso de redes sociales como Facebook:

A partir de este momento, nuestra cobertura se centra en coronavirus y empezamos a presentarle a la audiencia distintas maneras, con distintos formatos nuestras actualizaciones de información, tanto a través del NorteDigital.mx, como en la plataforma de FB, que hoy por hoy Norte Digital es líder de seguidores en FB con 1.2 millones (Entrevista Barranco).

\section{Los desafíos de la cobertura}

En este eje analítico se derivan problemáticas como el acceso a fuentes de información durante la cobertura COVID-19, la fuente oficial, y otra que cobró presencia de manera inesperada para los periodistas, la del personal médico que está en primera línea de atención a los pacientes. Otro elemento es la reflexión de los 
editores y reporteros sobre el papel y la función social del periodismo en contextos de emergencia.

\section{Las fuentes de información}

El primer acercamiento de los medios con la fuente oficial en el tema covid inició en el mes de enero, con conferencias presenciales dos veces por semana. A medida en que el avance de la epidemia urgió a las autoridades a proporcionar más información al respecto se hicieron diarias. Con el inicio de la fase 2 también cambió su modalidad a conferencias virtuales. La dinámica de estas conferencias fue detallada por varios de los entrevistados, en tanto todos los medios cubren la fuente oficial del gobierno del estado para el reporte diario de estadísticas:

Definitivamente la fuente más complicada y hostil es la fuente oficial. En Chihuahua el gobierno estatal centralizó la información y tiene el control de las cifras muy a modo. Organiza conferencias virtuales en las cuales las preguntas pasan por un filtro de manera que, si hay un cuestionamiento fuerte, esa pregunta no va a pasar (Entrevista Salcido).

En estas conferencias, los reporteros se conectan por medio de la plataforma Zoom y las preguntas se organizan a través de un grupo de WhatsApp. Entre los inconvenientes para el ejercicio periodístico está la interacción mediada por el coordinador de comunicación social del gobierno del estado. El intercambio entre reporteros y autoridades en las conferencias virtuales está controlado, no hay manera de interpelar directamente a los funcionarios a cargo, tampoco de garantizar que las preguntas de los reporteros se lean. En esta dinámica de intercambio selectivo de las preguntas coinciden todos los entrevistados.

La contraparte de las limitaciones en el trabajo con la fuente oficial se dio con el personal médico. Este sector ganó visibilidad cuando desde el mes de febrero protestaron por no tener la capacitación ni los insumos para enfrentar la emergencia sanitaria. Norte fue de los primeros medios en publicar fotos, videos y testimonios del personal de los hospitales del Instituto Mexicano del Seguro Social (IMSS) 6 y 66, habilitados por las autoridades para la atención de pacientes con COVID-19. La información que proporciona el personal médico representa un contrapeso a la información oficial: 
El personal médico o el personal de salud es muy importante para romper ese cerco informativo. Es importante porque de las propias áreas covid en los hospitales sale la información y sale porque tienen miedo. Cuando se empezó a acelerar los contagios, sobre todo enfermeras, empezaron a hablar (Entrevista Villagrana).

Otro tema de primer orden en la cobertura está en la industria maquiladora de Juárez, conformada por 345 empresas con un aproximado de 325 mil a 340 mil empleados. La industria maquiladora es el motor económico de la ciudad, según explica Cabrera, por los viajes al extranjero por parte de sus directivos se convirtió en un punto de contagio: "Estas empresas tienen ejecutivos que están viajando constantemente a sus corporativos que están en Asia, Estados Unidos, Canadá, por eso muchos de estos ejecutivos desgraciadamente llegaron contagiados a los centros de producción" (Entrevista Cabrera). El impacto de la CoviD-19 en la industria maquiladora al 28 de abril era de 13 personas fallecidas y entre 60 y 80 empresas en paros técnicos. Con el repunte de contagios en las maquiladoras, las autoridades empiezan a limitar la información sobre la estadística de contagios $\mathrm{y}$ fallecimientos en el sector.

\section{El rol del periodismo en el contexto de la emergencia sanitaria}

El trabajo de reporteo en plena contingencia sanitaria exige por parte de los periodistas algunas adaptaciones con el propósito de proteger su salud y cumplir con su labor informativa. El impedimento para verificar lo que sucede en hospitales o en lugares con brotes de contagio es visto como uno de los desafíos:

Para mí ha sido muy frustrante por el tema de la información, yo he cubierto salud durante todos estos años que tengo como reportera y cuando me reportaban un hecho en algún hospital, obviamente vas y te metes como si fueras paciente para traer información más fresca y cubrir bien el hecho (Entrevista Gamboa).

El distanciamiento en este sentido afecta la labor cotidiana de contacto y verificación directa de los periodistas. Por otra parte, los obliga a tomar otro tipo de medidas de protección para reducir el riesgo de contagio, sobre todo cuando tienen que entrevistar cara a cara como lo expresa el siguiente testimonio: 


\begin{abstract}
A la hora de recoger testimonios procuramos que no sea dentro de su casa, que sea afuera. Y que estemos a una distancia apropiada para que no ocurran contagios, incluso con personas que puedan ser de alto riesgo. Solamente así, incluso en ruedas de prensa que se han dado en asociaciones de funerarios, nos damos cuenta de que necesitamos mantener la distancia, pero no tenemos el equipo para grabar a distancia (Entrevista Villagrana).
\end{abstract}

Sin duda, un acontecimiento que influyó en el manejo informativo de los medios sobre la COVID-19 fue una nota publicada el 14 de abril por los periódicos El Diario de Juárez y El Diario de Chihuahua. La nota titulada "Prueban con fotos muertes por coviD" afirmaba que las autoridades estaban ocultando las cifras reales de los fallecimientos e incluía una fotografía donde se veían cadáveres acumulados en un cuarto de hospital, según los diarios había sido tomada en el IMSS de Juárez. Sin embargo, la escena correspondía a un hospital de Ecuador.

La respuesta del gobernador Javier Corral fue declarar ese mismo día que la nota publicada era una muestra del amarillismo de los periódicos aludidos, responsables de propagar el pánico moral entre la población. El incidente llegó a instancias federales en tanto la Secretaría de Gobernación anunció un procedimiento "administrativo sancionatorio" contra los dos periódicos por publicar información falsa pero, finalmente, desistieron. La reportera que firmó la nota se retractó en la edición del 15 de abril y El Diario de Juárez incluyó en su portada una nota aclaratoria en la que aseguraban haber recibido la información de buena fe, pero sin someterla a comprobación de autenticidad, por lo cual reconocían el error y ofrecían una disculpa a los lectores.

Las consecuencias de no verificar la información que se publica están presentes en entrevistas como la de Cabrera, quien refiere directamente el caso de El Diario de Juárez, por lo cual pusieron más atención en la verificación, ya que por la influencia de los medios, este tipo de información contribuye a generar pánico.

La cautela y los esfuerzos por comprobar la veracidad de los hechos noticiosos también aplica a la información que circula en redes sociales, desde testimonios o fotografías. Los reporteros aceptan que las redes sociales son un recurso más para generar información, porque circulan en tiempo real denuncias y situaciones que no se tocan por parte de la fuente oficial. En estos casos el tratamiento a este tipo de reporteo desde las redes consiste en contactar a los usuarios para verificar que no se trate de información falsa. 
Otro aspecto que aparece de manera recurrente en las entrevistas es la colaboración entre reporteros de distintos medios, como manera de triangular y confirmar información. Esta situación ya ha sido documentada por autores que trabajan el periodismo de desastres.

Yo he encontrado mucha solidaridad en el gremio, mucho apoyo en compañeros y compañeras de otros medios de comunicación. Incluso medios que estamos compitiendo. Ahora los compañeros están muy solidarios compartiendo toda esta información, especialmente aquella que tiene que ver con el coronavirus (Entrevista Barranco).

La reflexión sobre la función social del periodismo en el contexto de la pandemia apunta a los efectos preventivos de la información y al contraste de las cifras proporcionadas por la fuente oficial. Un aspecto interesante es que la información se valora a partir de la respuesta social que puede generar y también en tanto promueve el ejercicio ciudadano. El periodista aspira a que la información tenga sentido para las personas y los mueva a seguir las medidas que dictan los especialistas en salud.

\section{Los impactos de la cobertura}

El cambio y la adaptación de las rutinas de trabajo a las medidas de protección implicó para los reporteros un ajuste en sus dinámicas de reporteo. Desde la perspectiva editorial, la nueva realidad para el trabajo periodístico se vivió en los siguientes términos:

Obviamente los estamos cuidando y les estamos diciendo que traten de acercarse lo menos posible a los lugares donde están los focos de infección como pueden ser los hospitales o los lugares muy concurridos. Muchos de ellos tienen sus contactos y lo hacen a través de videollamadas, por teléfono, utilizando los chats como redes sociales, el WhatsApp, son las herramientas que utilizamos para poder conseguir la información (Entrevista Cabrera).

A medida que multiplicaban los casos positivos, el riesgo en la cobertura aumentaba para los reporteros. En Norte, por ejemplo, fue necesario hacerse cargo de la compra de equipos de protección, por lo demás, las medidas partían del conocimiento y experiencia de cada reporteo. 
En Ciudad Juárez los periodistas están habituados a lidiar con situaciones que implican algún tipo de riesgo a su actividad. En los últimos años, la violencia desatada por el crimen organizado a la cobertura informativa en contextos de violencia. Con ese antecedente, algunos entrevistados compararon la situación de la violencia y de la pandemia, su conclusión es que por primera vez experimentan una sensación de vulnerabilidad y riesgo que amenaza su vida:

En la pandemia está el peligro directamente con nosotros, nunca habíamos tenido ese peligro tan cercano. Nosotros como gremio y la población en general tenemos bastante experiencia en enfrentar la violencia, venga de donde venga, del narco, cualquier tipo de crimen organizado y sí tenemos experiencia en rolar en funerarias, áreas médicas, urgencias... No hay mayor reto que ejercer la profesión cuando la vida está en riesgo, con un enemigo que no se ve, que anda por ahí en el aire, entre las personas, en todos los lugares (Entrevista Villagrana).

En parte, la sensación de vulnerabilidad puede explicarse por la falta de seguridad y atención médica en situaciones de emergencia. Los entrevistados cuentan en su mayoría con afiliación al IMSS, y algunos con un seguro de vida en caso de fallecimiento. Sin embargo, en el contexto de la pandemia esperan otro tipo de garantías en caso de requerir atención médica. Un par de entrevistados manifestaron la posibilidad de contratar un seguro médico privado, aunque el tema de los bajos salarios lo vuelve poco factible. En todo caso, el tema de la pandemia problematiza las condiciones idóneas para garantizar la seguridad de los periodistas y con ello asegurar condiciones óptimas para su desempeño laboral.

Los impactos económicos de la pandemia no están definidos todavía. El riesgo de cierre de empresas y negocios está latente, a esta realidad no escapan los medios de comunicación. Para los periodistas este escenario acentúa la incertidumbre laboral que perciben ante la posibilidad de una reducción de salarios o directamente de despidos (Entrevista Salcido).

En las coberturas de emergencias y desastres, uno de los aspectos menos atendidos tiene que ver con el impacto emocional en los periodistas. Su exposición es equivalente a la de otros profesionales que atienden la emergencia en la primera línea. Por lo anterior no extraña que en las entrevistas manifiesten algunos de estos impactos emocionales derivados de su labor periodística: 
Yo no sé hasta qué punto me va a afectar todo lo que estamos viviendo en este momento porque nosotros lo recibimos todo, aunque no lo publiquemos. Pero no tenemos ningún filtro para afrontar las historias más crudas y esto sí tendrá un impacto en lo emocional. Algunos compañeros empiezan a manifestar insomnio y ansiedad (Entrevista Salcido).

En otros casos, entre la sugestión aparecen supuestos síntomas de coviD, como efecto de la sobreexposición a la información de la pandemia. Por otra parte, la fatiga de dar seguimiento pormenorizado a cada detalle e impacto de la cobertura resulta difícil de llevar para reporteros, quienes manifiestan cansancio y saturación de los contenidos covid.

En este escenario, las estrategias para reducir los impactos emocionales de la cobertura dependen del estilo personal de afrontar situaciones de crisis, por eso varían de un caso a otro. Algunos reporteros se acercan más al círculo de amistades y familiar: "Hay tres elementos que han sido fundamentales en mi caso particular. Tres elementos fundamentales para mantener el equilibrio emocional, que son la familia, la diversión y la terapia" (Entrevista Barranco).

En otros casos toman distancia informativa con el tema covid con las pocas actividades de esparcimiento que se pueden mantener, como la lectura. Un hecho curioso es narrado por un entrevistado que habla del humor como válvula de escape a la realidad de la epidemia: "Hay compañeros del medio o amigos cercanos que empezamos a hacer humor negro de la pandemia, pudiera ser cruel, pero es terapéutico para estos casos. Hacemos humor negro de los agentes funerarios, que llegan a los hospitales con equipos inadecuados, con un paliacate en la boca, un enterrador con un traje de bombero. Empiezan a surgir chistes, memes, risas, es humor negro y yo creo que es una forma de escaparse un rato de la realidad" (Entrevista Villagrana).

Lo extenuante de la cobertura de COVID-19 obliga a replantear esquemas de trabajo para evitar la saturación y su efecto en elevar niveles de ansiedad en los reporteros. Asimismo, considerar el apoyo psicológico para el manejo de estrés y ansiedad en la cobertura de emergencias sanitarias. 


\section{DISCUSIÓN}

\section{La experiencia de los periodistas juarenses en la cobertura de covid-19}

La crisis sanitaria por CoviD-19 en Ciudad Juárez obligó a los responsables editoriales de medios como El Heraldo de Juárez y Norte Digital a implementar estrategias de trabajo a distancia. En esta vía, el papel de las nuevas tecnologías fue fundamental para mantener la comunicación entre editores y reporteros, lo mismo que replicar dinámicas de trabajo por vía remota, como las conferencias por Zoom o bien el uso de las herramientas de Google. A diferencia del medio digital, en el periódico impreso esta modalidad permitió agilizar los tiempos de cierre para la edición. En el escenario pospandemia, el medio evaluará la pertinencia de mantener esta forma de trabajo para hacer frente a la crisis que enfrentan y reducir costos de renta de edificios e instalaciones. Es posible que uno de los efectos de la crisis sanitaria modifiqué las relaciones y dinámicas laborales.

Uno de los mayores desafíos de la emergencia se presentó en el trabajo de los reporteros. El acceso a la fuente oficial, mediado por plataformas, afectó, a decir de los entrevistados, la posibilidad de replicar y plantear directamente las preguntas a las autoridades de salud, quienes según la lectura de los reporteros manejan en sus informes cifras a modo que ocultan la profundidad de la crisis. Esta limitante encontró un contrapeso en la disposición del personal médico para exponer la situación de atención hospitalaria y contrastar así la versión de las autoridades.

Para los reporteros, la cobertura supuso un desafío en términos del acceso a fuentes y de verificación de datos. En las entrevistas se reconoce una práctica de intercambio y apoyo entre periodistas de diferentes medios para triangular versiones y evitar la difusión de información falsa que pueda comprometer al medio responsable. Este tipo de colaboración ya ha sido documentada en situaciones de desastres o emergencias.

El papel del periodismo en el contexto de la pandemia es altamente valorado por los reporteros. En su conjunto coinciden en dar a conocer información verificada y equilibrada de los actores involucrados. En sus respuestas está presente el papel social del periodismo como detonante de conductas que contribuyan a contener la epidemia y seguir las recomendaciones de las autoridades en la materia.

Uno de los aspectos más preocupantes tiene que ver con la percepción de vulnerabilidad que expresan los entrevistados. En su mayoría hacen referencia a 
coberturas que en otro momento implicaron algún tipo de riesgo, como las de los feminicidios y de la presencia del crimen organizado en la ciudad. Con la crisis sanitaria, expresan que por primera vez perciben una amenaza directa a su vida, en caso de resultar contagiados. Esta percepción parte de estar en contacto con familiares de enfermos, con personal médico y con el acercamiento necesario a sitios de alto riesgo como hospitales y funerarias.

Sin protocolos de protección, los reporteros se encargan directamente de adquirir insumos para protegerse como mascarillas, gel, guantes, en pocos casos los proporciona la empresa. Por otra parte, no todos cuentan con atención médica como parte de sus prestaciones laborales. Ante el riesgo de contagio, los entrevistados manifestaron que los periodistas deberían de contar con un servicio médico privado.

Por último, los impactos emocionales son ampliamente reconocidos por los entrevistados y documentados en la literatura sobre el tema. En el momento en el que se realizaron las entrevistas, los primeros indicios de ansiedad, insomnio y estrés se hacían presentes, también los rumores de contagios de compañeros de otros medios. La manera de enfrentar en lo emocional estos impactos dependía de manera absoluta de las iniciativas y los recursos individuales de cada periodista, desde el apoyo familiar, la terapia, o algunas formas de entretenimiento. Este aspecto es probablemente uno de los más invisibilizados en este tema.

Las exigencias de no perder el hilo de la cobertura también impactan de manera negativa en la salud emocional de los reporteros, quienes dan cuenta de una sobresaturación de contenidos, un cansancio y fatiga por estar al tanto de cada detalle que tenga relación con la emergencia sanitaria. En ese sentido, los primeros afectados por el exceso de información de la pandemia son los propios periodistas.

Explorar desde las prácticas de los periodistas los desafíos que enfrentan en la cobertura de desastres permitirá contar con elementos que permitan resolver de mejor forma este tipo de episodios, desde las etapas de planeación editorial hasta la atención de los impactos emocionales provocados por este tipo de coberturas.

\section{CONCLUSIONES}

La cobertura informativa de emergencias y desastres naturales exige a los periodistas una profesionalización orientada a la prevención y seguimiento de los 
acontecimientos de ese tipo. En condiciones que no son las óptimas por temas de salario y capacitación, presentan condiciones de vulnerabilidad ante los contextos de emergencia.

Desde el periodismo local, los casos de El Heraldo de Juárez y Norte Digital nos permitieron profundizar en el ejercicio periodístico en el contexto de la pandemia por CoviD-19. A pesar de que la literatura sobre el tema acentúa la necesitad de colaboración entre autoridades, periodistas y expertos, en el caso de Ciudad Juárez lo que privó fue la resistencia de las autoridades del estado para mantener cercanía y estar atentos a los cuestionamientos de los periodistas para una mejor comunicación del avance de la crisis sanitaria y las medidas para su contención.

Ante el acceso limitado con las autoridades del estado, el personal médico se reveló como una fuente de primer orden para triangular y contrastar la información oficial. En episodios de crisis sanitaria, el personal médico se convierte en una fuente de información valiosa, pero en el contexto de la pandemia por Covid-19 también supone desafíos para la verificación de la información proporcionada, como sucedió con el caso de El Diario de Juárez, el cual determinó la importancia que los medios locales le otorgan a la verificación.

Los periodistas juarenses entienden el valor de la información en la medida en la que ésta movilice conductas de prevención y seguimiento de las recomendaciones de los expertos, lo mismo en el contraste de la información proporcionada por la fuente oficial. El papel del periodismo en contextos de emergencia lo asumen como prioritario para dar voz a los afectados y difundir medidas que contribuyan a superar el episodio.

Finalmente, en las entrevistas quedó expuesta la situación de vulnerabilidad de los periodistas al no contar con una capacitación orientada a su protección en caso de contagio. Otro rubro delicado es el no tener acceso a atención médica de calidad, lo cual los lleva a considerar la contratación de un servicio médico pagado por ellos mismos. Al parecer y pese a la situación complicada por la que atraviesan los medios de comunicación en México, es necesario replantear la necesidad de invertir en la capacitación del personal y proporcionar condiciones para garantizar su salud e integridad en las coberturas con un grado mayor de riesgo.

En este estudio que recoge de manera preliminar, porque la emergencia por Covid-19 no ha concluido, los testimonios sobre los desafíos de los periodistas, sus miedos y preocupaciones en situaciones de emergencia, puede dar la pauta para que en conjunto, autoridades y dueños de los medios de comunicación, revaloren la importancia del trabajo de los reporteros en contextos de emergencia y 
contribuyan en la medida de lo posible a proporcionar mejores condiciones para que el periodismo cumpla con su función social en estas situaciones.

\section{REFERENCIAS}

Berkowitz, D. (1992). Non-routine news and newswork: Exploring a What-a-Story.Journal of Comunication, 42(1), 82-94. https://doi.org/10.1111/j.1460-2466.1992.tb00770.x

Bernabé, J. (2007). Orígenes, principios, objetivos y herramientas del periodismo preventivo. (Bernabé, Javier, ed.). Periodismo preventivo. Otras maneras de informar sobre las crisis y los conflictos internacionales (pp.17-33). Madrid, Catarata.

Camps, S. (1999). Periodismo sobre catástrofes. México, Ediciones Paulinas.

Checa, A. (2008). Ciudad Juárez: feminicidios, sociedad y medios (Checa Godoy y M. Ramírez Alvarado, eds.). Visiones de América: Comunicación, mujer e interculturalidad (pp. 193-211). Netbiblo.

Domínguez-Panamá, J. J. (2017). El periodismo de desastre: de las no-rutinas a las funciones sociales del periodista. Comhumanitas: revista científica de comunicación, 8(1). https://doi.org/10.31207/rch.v10i2.206

Hight, J., \& Smyth, F. (20 03). Tragedias \& Periodistas. Guía para una cobertura más eficaz. Dart Center for Journalism/Trauma and Columbia University.

Márquez, M., \& Sallie, H. (2016). Panorama de los perfiles demográficos, laborales y profesionales de los periodistas en México. Global Media Journal, 14(26).

Olsson, E. (2010). Defining crisis news events. Nordicom Review, 31(1). https://doi. org/10.1515/nor-2017-0122

Oyandel, R., \& Alarcón C. (2010). Reflexiones y desafíos: Una mirada al tratamiento televisivo de la catástrofe. Cuadernos.info, (26), 115-122. https://doi.org/10.7764/cdi.26.16

Reyna, A. (1998). Algunas contribuciones de la demografía al estudio de los desastres. (Garza Salinas, M. \& D. Rodríguez, coords.). Los desastres en México. Una perspectiva multidisciplinaria (pp.39-58). México, UNAM/UIA/UAM-X.

Rodríguez, P., \& Odriozola, B. (2010). Catástrofes y periodismo: el relato, los escenarios, las interacciones y las necesidades prácticas y psicológicas de todos los implicados. Estudios sobre el Mensaje Periodístico,18(2). https://doi.org/10.5209/rev_esmp.2012. v18.n2.41033

Rodríguez, R., \& Martín M. (2003). Periodismo de catástrofes: el 11 de septiembre. Análisis del suceso y experiencias vividas. Ámbitos, (9-10). https://doi.org/10.12795/ambitos.2003.i09-10.30

Segob \& Cenapred. (2006). Conceptos básicos sobre peligros, riesgos y su representación geográfica. 
Shih, T., Wijaya, R., \& Brossard, D. (2008). Media Coverage of Public Health Epidemics: Linking Framing and Issue Attention Cycle Towards an Integrated Theory of Print News Coverage of Epidemics. Mass Communication \& Society, 11(2). https://doi. org/10.1080/15205430701668121

Toledano, S., \& Ardèvol-Abreu, A. (2013). Los medios ante las catástrofes y crisis humanitarias: propuestas para una función social del periodismo. Communication \& Society/ Comunicación y Sociedad, 26(3).

Waisbord, S. (2010). Cuando la salud es titular: dengue, gripe AH1N1 y ciclos 'mediático-epidémicos'. Folios, 23. 